\title{
GATA4 protects against hyperglycemia-induced endothelial dysfunction by regulating NOX4 transcription
}

\author{
HONGFEI XU ${ }^{1 *}$, ZHEN WANG $^{2 *}$, ZEWEI SUN $^{2}$, YIMING NI $^{1}$ and LIANGRONG ZHENG ${ }^{2}$ \\ Departments of ${ }^{1}$ Cardiothoracic Surgery and ${ }^{2}$ Cardiology, The First Affiliated Hospital, \\ College of Medicine, Zhejiang University, Hangzhou, Zhejiang 310003, P.R. China
}

Received October 5, 2016; Accepted July 6, 2017

DOI: $10.3892 / \mathrm{mmr} .2017 .8062$

\begin{abstract}
Endothelial dysfunction is one of the most common complications associated with diabetes and may lead to atherosclerosis. Conflicting reports indicate that NADPH oxidase 4 (NOX4) induces hydrogen peroxide production and cytotoxicity, but also has a protective effect on endothelial dysfunction. The present study aimed to identify the transcription factor responsible for NOX4 expression using a transcription factor activation profiling plate array and chromatin immunoprecipitation. Data from these analyses indicated that GATA-binding protein 4 (GATA4) was able to mediate NOX4 transcription and is downregulated in human umbilical vein endothelial cells (HUVECs) that were exposed to hyperglycemic conditions as well as in the endothelial cells of a mouse diabetes model. Overexpression of GATA4 was demonstrated to lead to increased expression of NOX4 mRNA and protein. Furthermore, GATA4 overexpression resulted in increased nitric oxide (NO) production through the upregulation of endothelial NO synthase phosphorylation. Treatment with simvastatin, a drug known to preserve endothelial function through an unknown mechanism, improved endothelial cell function by upregulating GATA4 expression in HUVECs exposed to hyperglycemia. Results from these experiments demonstrated that GATA4 may inhibit diabetes-induced endothelial dysfunction by acting as a transcription factor for NOX4 expression and increasing NO production. Thus, the present study revealed a novel molecular mechanism underlying endothelial dysfunction in diabetes and identified GATA4 as a potential therapeutic target.
\end{abstract}

Correspondence to: Dr Liangrong Zheng, Department of Cardiology, The First Affiliated Hospital, College of Medicine, Zhejiang University, 79 Qingchun Road, Hangzhou, Zhejiang 310003, P.R. China

E-mail: zlrylnn@126.com

${ }^{*}$ Contributed equally

Key words: GATA-binding protein 4, NADPH oxidase 4, endothelial dysfunction

\section{Introduction}

Cardiovascular complications are among the most common complications associated with diabetes mellitus and are a major cause of hospital admission and mortality among patients with diabetes $(1,2)$. Atherosclerosis is a cardiovascular condition experienced by many patients with diabetes and can lead to severe consequences, such as myocardial infarction, ischemic stroke and ischemia of peripheral tissues $(3,4)$. Endothelial dysfunction, which results in the disruption of the balance between vasodilation and vasoconstriction, is an initiating factor in the development of atherosclerosis $(5,6)$. Thus, a therapeutic strategy for restoring endothelial function would be valuable in preventing serious cardiovascular complications in patients with diabetes.

Previous studies on NADPH oxidase 4 (NOX4), an isoform of the NOX family of enzyme complexes, reported conflicting harmful and beneficial results. The main function of NOX4 results in the production of hydrogen peroxide (7), which indicated that overactivation and induction of NOX4 expression may lead to the development of vascular diseases. Indeed, several studies have demonstrated that NOX4 activity can be harmful; for example, one study investigated the effects of NOX4 knockout in a mouse model of stroke and revealed that NOX4-knockout mice exhibited less oxidative stress, blood-brain barrier leakage and neuronal apoptosis compared with control mice (8). Another study reported that cardiac-specific deletion of NOX4 inhibited cardiac hypertrophy, fibrosis and apoptosis in response to pressure overload (9). By contrast, several studies have demonstrated that NOX4 activity is beneficial in cardiovascular diseases. For example, overexpression of NOX4 was reported to enhance vasodilatation and reduce blood pressure (10). In addition, NOX4 expression was revealed to inhibit the development of endothelial dysfunction and atherosclerosis in mice deficient in low-density lipoprotein receptor (7). These inconsistent results regarding the role of NOX4 in cardiovascular disease indicated that a more comprehensive understanding of NOX4 signaling is required for the development of treatments to correct endothelial dysfunction through the regulation of NOX4 activity.

In the present study, a promoter-binding transcription factor activation profiling plate array and chromatin immunoprecipitation (ChIP) were used to identify GATA-binding protein 
4 (GATA4) as the transcription factor that may be responsible for regulating NOX4 expression in human umbilical vein endothelial cells (HUVECs). Subsequently, the expression levels of GATA4 and NOX4 in response to hyperglycemia were investigated, as well as the effects of GATA4 overexpression in HUVECs. Finally, whether simvastatin treatment was able to improve endothelial function by upregulating GATA4 was examined. The results of the present study may provide a novel mechanism of NOX4 regulation as well as a new target for treating endothelial dysfunction in patients with diabetes.

\section{Materials and methods}

Cell culture. HUVECs were purchased from ScienCell Research Laboratories, Inc. (San Diego, CA, USA) and grown in Endothelial Cell Medium (ECM; 5.5 mM glucose; ScienCell) at $37^{\circ} \mathrm{C}$. Hyperglycemia was induced by growing cells in DMEM (Thermo Fisher Scientific, Inc., Waltham, $\mathrm{MA}, \mathrm{USA}$ ) at $37^{\circ} \mathrm{C}$ for $12 \mathrm{~h}$ (serum starvation), followed by exposure to $33 \mathrm{mM}$ glucose for $72 \mathrm{~h}$. Control cells were cultured in normal ECM containing $5.5 \mathrm{mM}$ glucose.

Mouse diabetes model. Male C57BL/6 mice were purchased from the Shanghai Laboratory Animal Center of the Chinese Academy of Sciences (Shanghai, China). All mice were 8 weeks old and weighed 25-30 g. Mice were housed in the animal facility, which was maintained at $20-25^{\circ} \mathrm{C}$ with $55 \%$ relative humidity and an automatic $12 \mathrm{~h}$ light/dark cycle. All mice received a standard laboratory diet and tap water ad libitum, and were acclimated for 1 week prior to experimentation. A total of 40 mice were used in this experiment; 20 in the control group and 20 in the diabetes group. Mice received an intraperitoneal injection of $110 \mathrm{mg} / \mathrm{kg}$ streptozotocin (STZ; Sigma-Aldrich; Merck KGaA, Darmstadt, Germany) to induce the diabetes model. Blood glucose concentration was measured at $72 \mathrm{~h}$ following STZ injection. Mice with a glucose level $\geq 16.7 \mathrm{mmol} / \mathrm{l}$ were considered diabetic. The mice in the control group received an intraperitoneal injection of saline. This study was approved by the Ethics Committee of The First Affiliated Hospital, College of Medicine, Zhejiang University (Hangzhou, Chin).

Promoter-binding transcription factor profiling plate array. The promoter-binding Transcription Factor Profiling Plate Array (Signosis, Inc., Santa Clara, CA, USA) was performed according to the manufacturer's protocol. Briefly, nuclear proteins were extracted using the nuclear protein extraction kit provided with the array, according to the manufacturer's instructions. Biotin-labeled transcription factor probes (Signosis, Inc.) were mixed with $10 \mu$ l nuclear extract with or without NOX4 promoter $(1.15 \mu \mathrm{g})$, and the complex was incubated at $25^{\circ} \mathrm{C}$ for $30 \mathrm{~min}$. Subsequently, transcription factor-DNA complexes were separated from unbound probes using the isolation columns provided in the kit. The bound probes were eluted from the columns with elution buffer, and hybridization of the eluted probes was performed with the hybridization plates. Finally, the bound probes were detected by streptavidin-horseradish peroxidase conjugation using a microplate fluorometer. In this assay, if the promoter sequence contains a transcription factor-binding site, it will compete with the biotin-labeled probe, leading to detection of a lower signal. The promoter sequence was kindly provided by Professor Adrian Manea (Institute of Cellular Biology and Pathology 'Nicolae Simionescu', Bucharest, Romania) as described previously $(11,12)$. The promoter sequence was as follows: 5'-ATATCCTATGGCCTGTGTTGTAAGATTT TTTATTTTAAGAGTTTAAAAGCCATTCGATTAAGCA ACTAGTAGTAAACTTTTCTGTCTCTCATTAGTTGTT AAATCTAGAAAAGTTATTGGTTTAACAAACCTTTATT GGATATCTATTTTGCATCAGATTTTTTTCTACATGA ATGACATTTTTTTCTCTCTCTTTTCTAATACTGATT TGTTGCAGTGTGATTGAGAGGATTCCTATTACTTACA AACAGACTAGGTATTAAAACAAAAAGATTCCCGAAT AGAGTGAACCTAACCTAGAGCCCCTAAGAATTACATC AGCTTTAACCATTAATTCCCTGGGTTCTTCTTATTCA GTAATGTCTTAAAACCTTTTTATCAGTATGGGATGG GCGGGCAAGGGGATAAAGAAACTGGCGGCTGGT GGGAAGCCTTAGGTAAGGCTGTCAGGGGGGCTT TAGTTTGGGAGTGGGATAATTTGCTGAGGAAGGGTG GGAGAAACGTGAACTAGCACACAAAAGGCTGTTTCC GGCTCAAATTTCGTTACCAAGGCTCTTTGAGATGAA CTTTTTTGGGGGAAAACAATCAGTCTAAAAGAGCTG TGTCTTCTTGTGTGTGTGTGTGTGTGTGTGTGTGT GTGTGTGTGTGTACAAGGGGGCGGCGAGGGTCCCCA CTTTTAGTATGAGTAGCATTGTTCACATGTTTGCCA GTATTTTGGAGCCTGGCAGGCCTGGGTAGAGGCT GCGGGGGACGCCTCCAAGTTCCCACCCGGGACATCC TGAACAGCAGCAGCCACAACAACAGGCTCGCCCC TAGACAAAGGGGCCGGCGCGGCGGAGCAGACTGGT GCAGCCTGGGCCGCGCGCTCAGAGCGCTGGGCGTCT GGGCAGCTGAGTGGGCAGAGCTGACCCGGTGCGG GTGGGAGTCAGGGCGCCCGGAAAACCCGGCTCT GGGTAGCAGACCCCGCCCGGGCTGGCTCGGCGCCG GGCCTTCGGGCTTCCACTCAGTCTTTGACCCTCGGT CCTCGCTCAGCGGCCCGGCAGGCCGCACAACTGTAA CCGCTGCCCCGGCCGCCGCCCGCTCCTTCTCGGTCC GGCGGGCACAGAGCGCAGCGCGGCGGGGCCGGCG GCATGGCTGTGTCCTGGAGGAGCTGGCTCGCCAAC GAAGGGGTTAAACACCT-3'.

ChIP. ChIP was performed using the SimpleChIP kit from Cell Signaling Technology, Inc. (Danvers, MA, USA), according to the manufacturer's protocol. Briefly, owing to the lack of a ChIP-grade GATA4 antibody, HUVECs were cultured in 6-well plates until they reached $60-80 \%$ confluence and transfected with GATA4-Flag $(2 \mu \mathrm{g})$ or Flag-only overexpression plasmid (2 $\mu \mathrm{g}$; Vigene Biosciences, Inc., Rockville, MD, USA). Transfection was performed using jetPEI-HUVEC transfection reagent (Polyplus-Transfection SA, Illkirch, France) according to the manufacturer's protocol. Cells were transfected for $4 \mathrm{~h}$ at $37^{\circ} \mathrm{C}$, after which the transfection medium was replaced with growth medium containing 30\% fetal bovine serum (Sigma-Aldrich; Merck $\mathrm{KGaA}$ ) and antibiotics. Following transfection, HUVECs were fixed in $1 \%$ formaldehyde in PBS at $37^{\circ} \mathrm{C}$ for $10 \mathrm{~min}$. Chromatin was digested with the addition of $5 \mu 1$ nuclease at $37^{\circ} \mathrm{C}$ for $20 \mathrm{~min}$, and DNA fragments of 200-800 bp were obtained. Protein A/G agarose was used for preclearing at $4^{\circ} \mathrm{C}$ for $1 \mathrm{~h}$ prior to incubation with $10 \mu \mathrm{g}$ of anti-Flag (Abcam, Cambridge, MA, USA) or the immunoglobulin G 
(included in kit) antibody at $4{ }^{\circ} \mathrm{C}$ overnight with rotation. The resultant complex was incubated in $5 \mathrm{M} \mathrm{NaCl}$ and $20 \mathrm{mg} / \mathrm{ml}$ proteinase K solution (Cell Signaling Technology, Inc.) at $65^{\circ} \mathrm{C}$ for $2 \mathrm{~h}$ for the reversal of crosslinking. Finally, the DNA was purified using spin columns and subjected to real-time polymerase chain reaction (PCR) analyses using SYBRPremix Ex TaqII (Tli RNase H Plus) from Takara Biotechnology Co., Ltd. (Dalian, China). The primers were as follows: forward TAAAAGCCATTCGATTAA, reverse GTCTGTTTGTAAGTAATAGGAA. The thermocycling conditions involved an initial denaturation at $95^{\circ} \mathrm{C}$ for $30 \mathrm{sec}$, followed by 34 cycles of $95^{\circ} \mathrm{C}$ for $5 \mathrm{sec}$ and $60^{\circ} \mathrm{C}$ for $30 \mathrm{sec}$. PCR products were separated by $1 \%$ gel electrophoresis using agarose gels prestained with ethidium bromide. Bands were analyzed using Image J1.5.1 (National Institutes of Health, Bethesda, MD, USA).

Western blotting. Western blotting was performed as previously described (13). Briefly, protein was extracted from $1.5 \times 10^{6}$ cells, and the following antibodies were used: Anti-endothelial nitric oxide synthase (eNOS; ab76198; 1:1,000; Abcam), anti-phosphorylated (p)-eNOS (ab76199; 1:1,000; Abcam), anti-GATA4 (ab84593; 1:1,000; Abcam), anti-NOX4 (ab133303; 1:1,000; Abcam) and anti-GAPDH (ab8345; 1:1,000; Abcam) primary antibodies, and HRP-conjugated secondary antibodies [goat anti-rabbit (ab6721; 1:2,000; Abcam), goat anti-mouse (ab6789; 1:2,000; Abcam;)]. Image J version 1.5.1 (National Institutes of Health, Bethesda, MD, USA) was used to perform densitometric analysis.

Reverse transcription-quantitative PCR (RT-qPCR) analysis of NOX4 expression. RT-qPCR was conducted as previously described (13). Briefly, RNA was extracted from $1.5 \times 10^{6}$ cells using a total RNA purification kit (Beyotime Institute of Biotechnology, Haimen, China) and reverse transcription was performed using a PrimeScript 1st Strand cDNA Synthesis kit (Takara Biotechnology Co., Ltd.) according to the manufacturer's instructions. The primers used were as follows: $\beta$-actin, forward ATTGGCAATGAGCGGTTC, reverse GGATGC CACAGGACTCCAT; NOX4, forward CAGATGTTGGG GCTAGGATTG, reverse GAGTGTTCGGCACATGGGTA; GATA1, forward CTGTCCCCAATAGTGCTTATGG, reverse GAATAGGCTGCTGAATTGAGGG; GATA2, forward GCA ACCCCTACTATGCCAACC, reverse CAGTGGCGTCTT GGAGAAG; GATA3, forward GCCCCTCATTAAGCC CAAG, reverse TTGTGGTGGTCTGACAGTTCG; GATA4, forward CGACACCCCAATCTCGATATG, reverse GTT GCACAGATAGTGACCCGT; GATA5, forward CTTCGT GTCCGACTTCTTGGA, reverse CCGAGGCATTCCTTG TGGA; GATA6, forward CTCAGTTCCTACGCTTCGCAT, reverse GTCGAGGTCAGTGAACAGCA.

Immunohistochemistry. Immunohistochemical staining for GATA4 expression was performed as previously described $(13,14)$, using a primary antibody against GATA4 (ab84593; 1:100; Abcam). The endothelial layer of the thoracic aorta was sliced into 6-8 $\mu \mathrm{m}$ thick sections. Primary antibodies were detected using a HRP conjugated goat anti-rabbit secondary antibody was from (ab6721; 1:2,000; Abcam). Positive expression was assessed by determining the $\%$ of positive GATA4 staining in the entire visual field.

Nitric oxide (NO) measurement. NO levels in the supernatant of HUVEC cultures were measured using a NO Assay kit (Beyotime Institute of Biotechnology) as previously described (15). Briefly, the supernatant of endothelial cell medium was collected with a pipette. A standard curve was prepared from standard solutions of 2-50 $\mu \mathrm{mol} / 1$ nitrite in ECM. Samples of standard solutions or cell supernatants were reacted with nitrate reductase in a 96 -well plate for $40 \mathrm{~min}$ prior to the addition of Griess Reagents I and II. Samples were incubated at room temperature for $10 \mathrm{~min}$, and the absorbance in each well was measured at $540 \mathrm{~nm}$, using a microplate reader, to determine the nitrite concentrations.

Simvastatin treatment. HUVECs were incubated with $5.5 \mathrm{mM}$ (control) or $33 \mathrm{mM}$ (high) glucose with or without $1 \mu \mathrm{mol} / 1$ simvastatin (Sigma-Aldrich; Merck KGaA) for $72 \mathrm{~h}$ at $37^{\circ} \mathrm{C}$.

GATA4 knockdown. HUVECs were cultured in 6-well plates until they reached $60-80 \%$ confluence. Cells were transfected with GATA4 small interfering (si)RNA (sc-35455) or negative control (sc-37007) siRNA (Santa Cruz Biotechnology, Inc., Dallas, TX, USA) using Lipofectamine ${ }^{\circledR}$ RNAiMAX Transfection Reagent (Thermo Fisher Scientific, Inc.) according to the manufacturer's protocol. The cells were incubated at $37^{\circ} \mathrm{C}$ for 2 days. Experiments were performed $48 \mathrm{~h}$ following transfection.

Migration assay. The migration assay was performed in a Transwell chamber that was fitted with an $8 \mu \mathrm{m}$ pore size membrane filter (Corning Inc., Corning, NY, USA). Cells $\left(2 \times 10^{4}\right)$ were plated in the upper chamber in DMEM without FBS and incubated at $37^{\circ} \mathrm{C}$ for $24 \mathrm{~h}$. The lower chamber contained DMEM with $10 \%$ FBS. Following incubation, cells on the bottom surface of the filter were fixed and stained with Hoechst 33258 (Beyotime Institute of Biotechnology). The number of migrating cells was quantified by counting the total number of cells in 10 fields per chamber using a fluorescence microscope.

Statistical analysis. Data are expressed as the mean \pm standard deviation. All in vitro cellular preparations, experiments and measurements were repeated at least three times. Data from in vivo experiments represent the averages of at least eight measurements. Significant differences between groups were evaluated with an unpaired Student's t-test after the homogeneity of variance was confirmed with an F-test or one-way analysis of variance for more than two groups. $\mathrm{P}<0.05$ was considered to indicate a statistically significant difference.

\section{Results}

GATA4 is a transcription factor for NOX4. The promoter-binding transcription factor profiling plate array was used to identify the transcription factor that may be responsible for controlling NOX4 expression. The relative light units (RLU) for the GATA probe were increased for both the control HUVECs/(control HUVECs + NOX4 promoter) and hyperglycemia HUVECs/(hyperglycemia HUVECs + NOX4 promoter) 

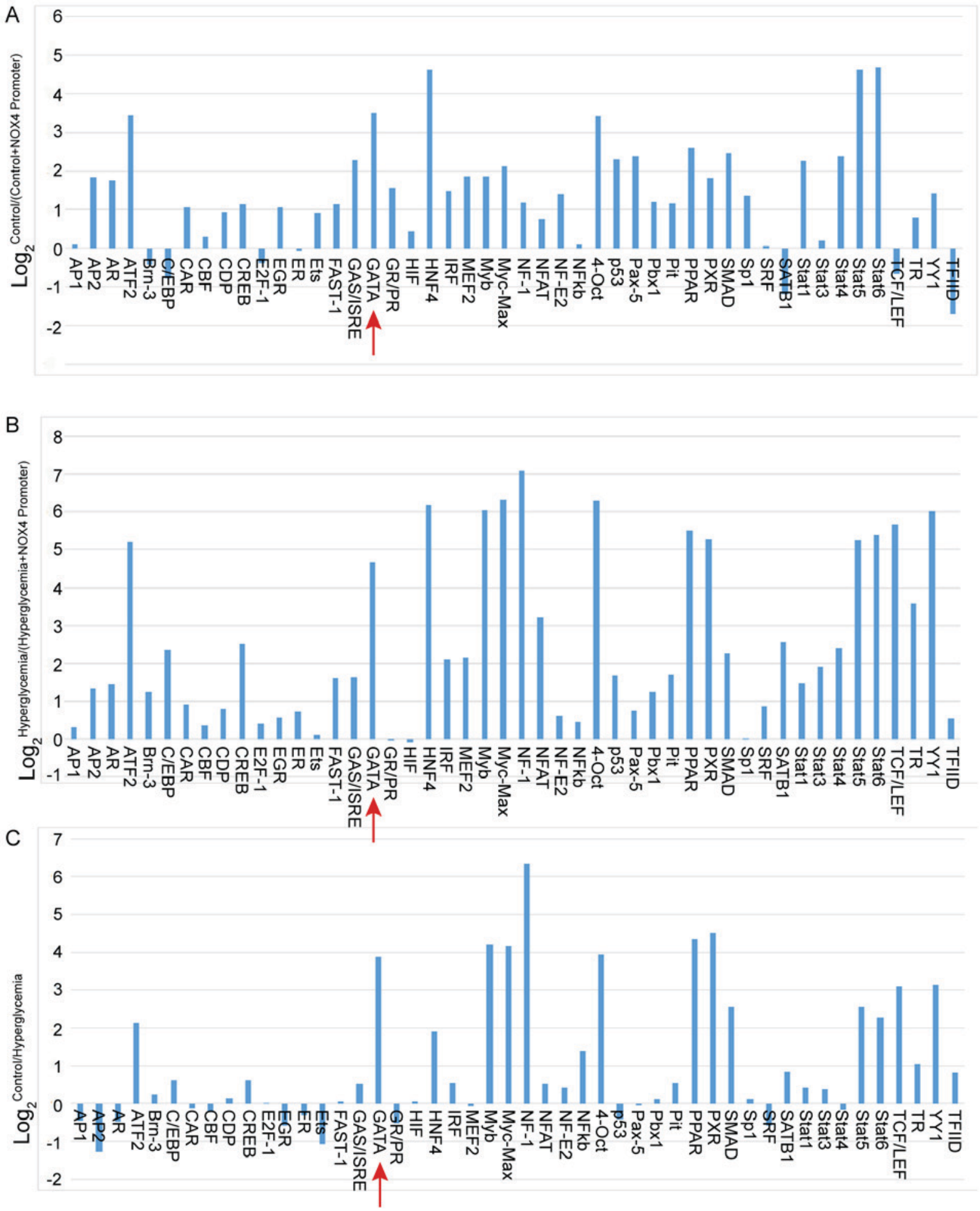

Figure 1. GATA is a transcription factor for NOX4 expression. A transcription factor binding screening assay was performed to identify the transcription factor of NOX4. (A) Comparison of GATA expression (in relative light units) in HUVECs cultured in normal glucose medium with or without NOX4 promoter. (B) Comparison of GATA expression in HUVECs cultured in high-glucose medium with or without NOX4 promoter. (C) Comparison of GATA expression in HUVECs cultured in control or high-glucose medium. GATA, GATA-binding protein; HUVEC, human umbilical vein endothelial cells; NOX4, NADPH oxidase 4 .

treatments (Fig. 1A and B, respectively), indicating that GATA protein can bind with the NOX4 promoter. Furthermore, the RLUs for the GATA probe were greater in HUVECs cultured in normal glucose medium compared with that in HUVECs cultured in high-glucose medium, indicating that GATA activity was inhibited by hyperglycemia treatment (Fig. 1C).
As this assay is not able to differentiate between the GATA proteins, the mRNA expression levels of GATA1-6 were measured in control or hyperglycemia-stimulated HUVECs.

As shown in Fig. 2A, hyperglycemia inhibited GATA4 transcription, whereas the mRNA expression of other GATA genes remained unchanged. Therefore, it was 
A

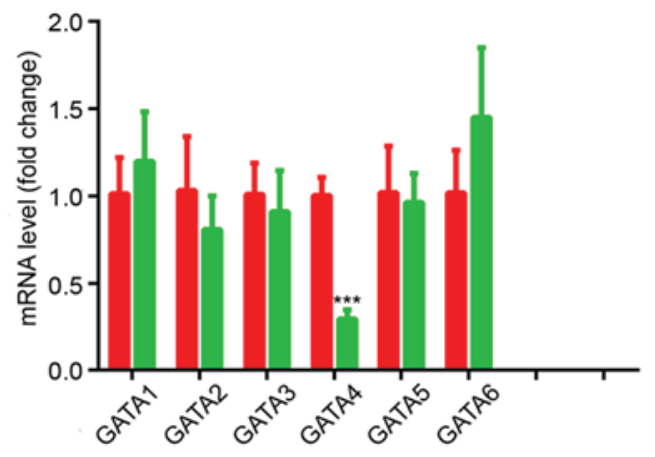

B

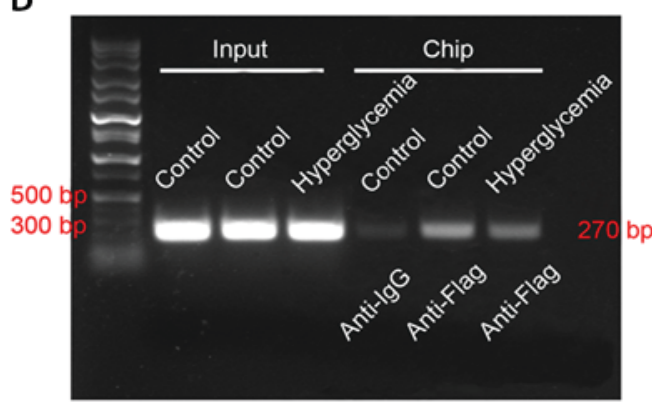

C

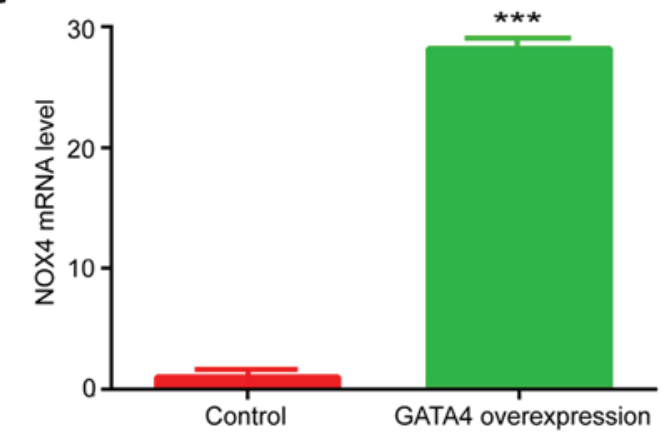

$\mathrm{D}$
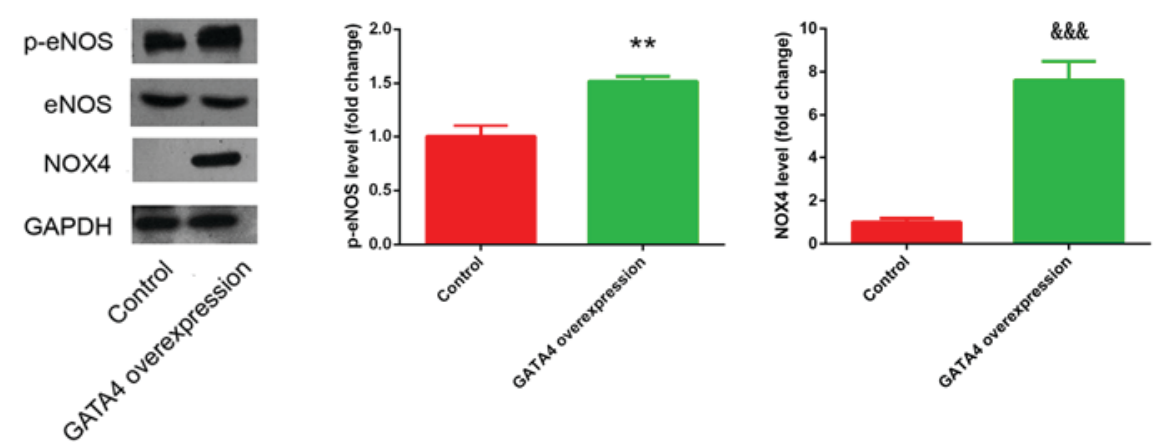

Figure 2. GATA4 mediates NOX4 expression. (A) GATA1-6 mRNA expression in HUVECs exposed to control or hyperglycemic conditions; ${ }^{* * *}$ P $<0.001$, hyperglycemia vs. control group. (B) Chromatin immunoprecipitation results for binding of GATA4 to the NOX4 promoter under normoglycemic control conditions and hyperglycemic conditions; ${ }^{\%} \% \% \mathrm{P}<0.001$, control anti-Flag vs. control anti-IgG group; ${ }^{\# \#} \mathrm{P}<0.01$, hyperglycemia anti-Flag vs. control anti-Flag group. (C) NOX4 mRNA expression upon GATA4 overexpression; ${ }^{* * *} \mathrm{P}<0.001$, GATA4 overexpression group vs. control. (D) NOX4 and p-eNOS protein expression in HUVECs overexpressing GATA4 were measured by western blot analysis; ${ }^{* *} \mathrm{P}<0.01$, GATA4 overexpression group vs. control; \&\&\& $<0.001$, GATA4 overexpression group vs. control. eNOS, endothelial nitric oxide synthase; GATA, GATA-binding protein; IgG, immunoglobulin G; NOX4, NADPH oxidase 4; p, phosphorylated.

concluded that GATA4 may be a transcription factor for NOX4.

These results were further confirmed by ChIP analysis (Fig. 2B). The ChIP band for the anti-Flag group exhibited increased intensity compared with the control group, confirming that GATA4 may be able to induce transcription of NOX4. In addition, the anti-Flag ChIP band corresponding to HUVECs exposed to hyperglycemia exhibited a lower intensity compared with the control group, indicating that hyperglycemia inhibited the binding of GATA4 to the NOX4 promoter. 
A

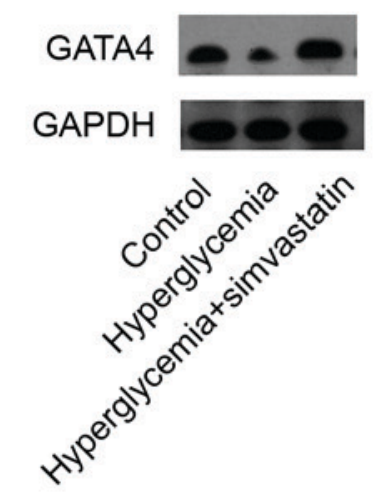

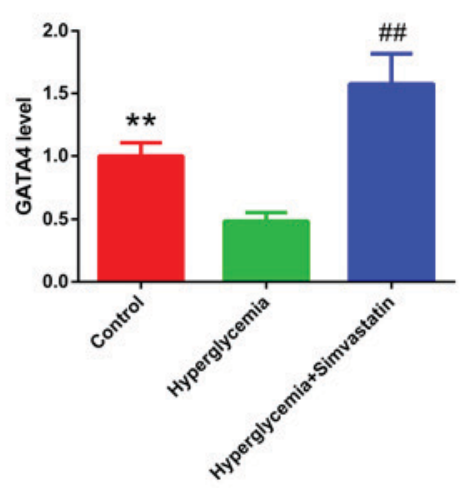

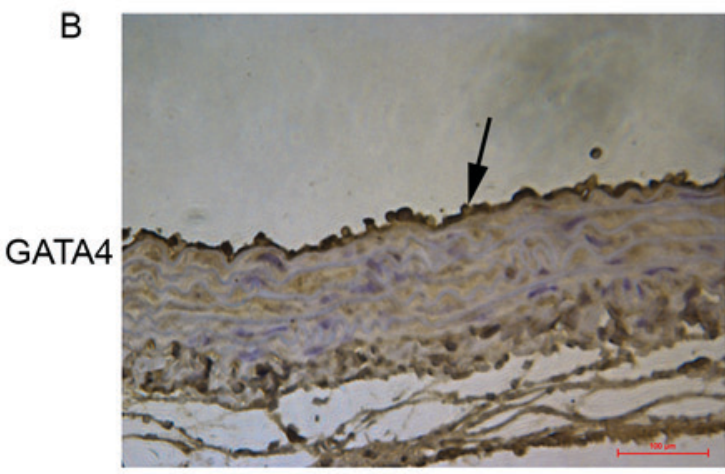

Control

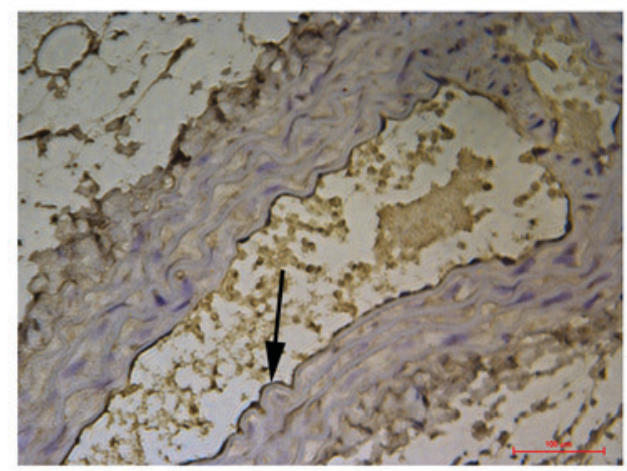

Diabetes

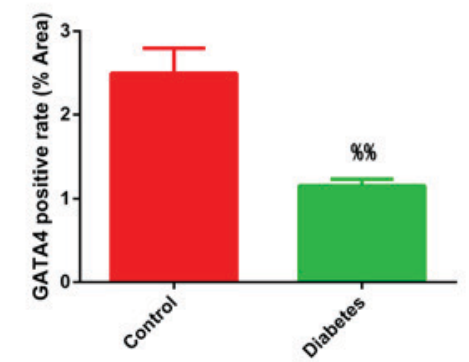

Figure 3. GATA4 protein expression is downregulated under hyperglycemic conditions. (A) Western blot analysis of GATA4 protein expression in HUVECs exposed to high-glucose medium with or without simvastatin treatment; ${ }^{* *} \mathrm{P}<0.01$, hyperglycemia vs. control group; ${ }^{\# \#} \mathrm{P}<0.01$, hyperglycemia + simvastatin vs. hyperglycemia group. (B) Immunohistochemical staining for GATA4 protein expression in endothelial cells (arrows) from a mouse diabetes model; ${ }^{\%} \mathrm{P}<0.01$, diabetes vs. control group.

NOX4 mRNA and protein expression levels were also analyzed in HUVECs that overexpressed GATA4 (Fig. 2C and D, respectively). GATA4 overexpression induced an increase in NOX4 mRNA and protein expression under normal glucose condition compared with the control cells. These experimental results confirmed that GATA4 is a transcription factor for NOX4.

GATA4 expression is downregulated in HUVECs exposed to hyperglycemic conditions. The expression of GATA4 protein in HUVECs cultured in control vs. high-glucose medium was measured by western blot. Hyperglycemia resulted in a decrease in GATA4 expression in HUVECs compared with untreated control cells (Fig. 3A). This finding was further confirmed in vivo using a mouse diabetes model, which exhibited lower expression of GATA4 in the endothelium of the thoracic aorta compared with healthy control mice (Fig. 3B).

GATA4 overexpression improves endothelial function by increasing NO levels. The role of GATA4 in endothelial function under hyperglycemic conditions was also investigated using HUVECs that overexpressed GATA4. GATA4overexpression resulted in an increase in the level of NO (Fig. 4A) as well as an increase in p-eNOS protein expression (Fig. 2D) in HUVECs stimulated with high-glucose medium, which indicated that GATA4 expression improved endothelial function under hyperglycemia.

GATA4 contributes to the protection against endothelial dysfunction achieved by simvastatin. A previous study has 
A

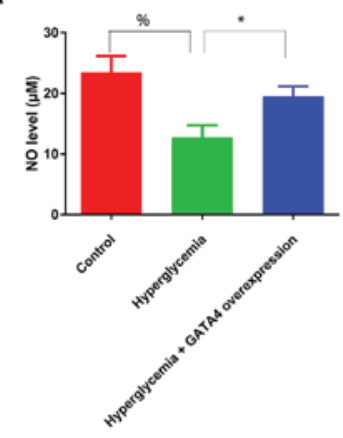

B
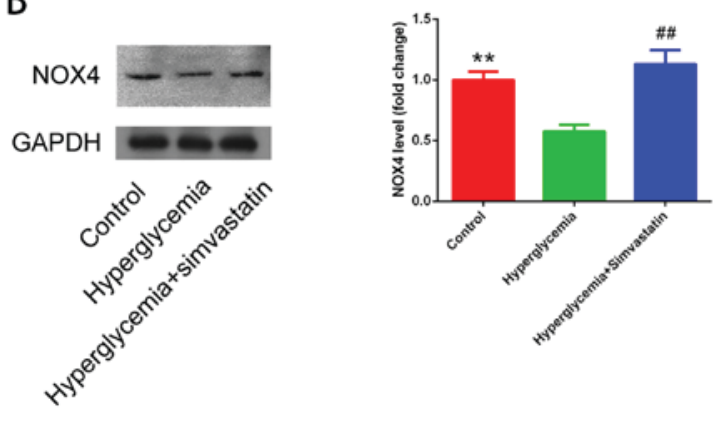

C

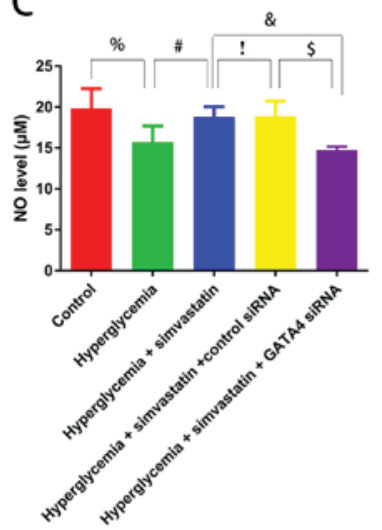

D

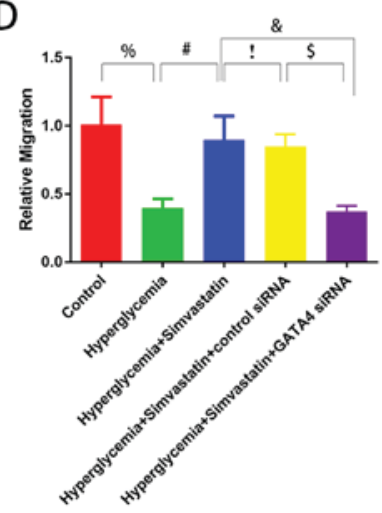

Figure 4. GATA4 improves endothelial function and is involved in protection of endothelial function by simvastatin. (A) NO levels in HUVECs with and without GATA4 overexpression. ${ }^{\%} \mathrm{P}<0.05$ control vs. hyperglycemia; ${ }^{\text {}} \mathrm{P}<0.01$ hyperglycaemia vs. hyperglycaemia + GATA4 overexpression. (B) Western blotting results demonstrating that NOX4 expression in HUVECs exposed to normoglycemic conditions as well as hyperglycemic conditions with or without simvastatin treatment; ${ }^{* *} \mathrm{P}<0.01$, hyperglycemia vs. control group; ${ }^{\# \#} \mathrm{P}<0.01$, hyperglycemia + simvastatin vs. hyperglycemia group. (C) NO levels in the supernatant of HUVECs exposed to control culture, hyperglycemia, hyperglycemia + simvastatin and hyperglycemia + simvastatin + GATA4-siRNA (D) Migration analysis in HUVECs exposed to control culture, hyperglycemia, hyperglycemia + simvastatin and hyperglycemia+simvastatin+GATA4-siRNA.(CandD) ${ }^{\%} \mathrm{P}<0.05$, hyperglycemia vs.control group; ${ }^{\#} \mathrm{P}<0.05$, hyperglycemia + simvastatin vs. hyperglycemiagroup 'P $>0.05$ hyperglycemia + simvastatin vs. hyperglycemia + simvastatin + control siRNA; ${ }^{\text {P}} \mathrm{P}<0.05$ hyperglycemia + simvastatin + control siRNA vs. hyperglycemia + simvastatin + GATA4-siRNA; ${ }^{\&} \mathrm{P}<0.05$, hyperglycemia + simvastatin + GATA4-siRNA vs. hyperglycemia + simvastatin group. GATA4, GATA-binding protein 4; HUVECs, human umbilical vein endothelial cells; NO, nitric oxide; NOX4, NADPH oxidase 4; siRNA, small interfering RNA.

demonstrated that simvastatin treatment functions to protect endothelial function in diabetes (16). Therefore, whether

this effect was achieved through GATA4 expression was investigated in HUVECs. Cells treated with simvastatin exhibited increased protein expression levels of GATA4 (Fig. 3A) and NOX4 (Fig. 4B), as well as increased levels NO (Fig. 4C) in hyperglycemia-stimulated HUVECs. Conversely, GATA4-siRNA treatment decreased the protective effects of simvastatin by inhibiting NO production (Fig. 4C).

To further confirm the protective role of GATA4 in endothelial dysfunction, a migration assay was performed. Simvastatin treatment resulted in increased cell migration in hyperglycemia-stimulated HUVECs, whereas GATA4 knockdown abolished this effect (Fig. 4D). These findings demonstrated the role of GATA4 in protecting endothelial function in hyperglycemia and indicated that the effects of simvastatin may be achieved through GATA4.

\section{Discussion}

The results of the present study indicated that GATA4 was able to regulate the transcription of NOX4. This result is supported by previous studies that demonstrated an association between GATA4 and NOX4. For example, Murray et al observed that activation of GATA4 transcription was dependent on NOX4 activation (17). The present results indicated that GATA4 was able to induce transcription of NOX4, which indicated that positive feedback occurs between GATA4 and NOX4. Another study reported that CCAAT enhancer-binding protein (C/EBP) induces transcription of NOX4 (11), whereas other studies demonstrated an interaction between GATA4 and C/EBP $(18,19)$, which indicated that GATA4 may serve a role in the regulation of NOX4 expression.

The present study demonstrated GATA4 had a protective effect against endothelial dysfunction induced by hyperglycemia. First, GATA4 expression was inhibited in HUVECs exposed to high-glucose treatment. This result was consistent with the results of previous reports that demonstrated that cardiac GATA expression is downregulated in diabetic mice $(20,21)$. The decreased GATA4 expression observed in the present study further indicates that GATA4 may be associated with the molecular mechanisms underlying diabetes. In addition, genetics studies have reported that GATA4 mutations may cause neonatal and childhood-onset diabetes (22), and that GATA4 polymorphism is associated with diabetes in adults (23). Second, as NOX4 was revealed to protect against endothelial dysfunction in diabetes, GATA4 presumably should have a similar effect given that GATA4 induces NOX4 transcription. Third, GATA4 has previously been reported to have the ability to inhibit apoptosis $(24,25)$. As endothelial cell apoptosis is a cause of endothelial dysfunction (26), the present study proposed that the protective effect of GATA4 overexpression may be due to the inhibition of endothelial cell apoptosis stimulated by hyperglycemia. Fourth, in the presence of diabetes, GATA4 has been revealed to serve a protective role in many other cardiovascular diseases. For example, GATA4 overexpression was reported to support cardiac adaptive responses and survival, whereas GATA4 ablation induced cardiomyocyte apoptosis and heart dysfunction (27). In addition, GATA4 was demonstrated to protect cardiomyocytes from doxorubicin-induced cell death (28). 
The present study had several limitations. First, the co-factors required for GATA4-induced NOX4 transcription have yet to be identified. Second, although GATA4 protected HUVECs against hyperglycemia-induced endothelial dysfunction by enhancing NO secretion, this effect requires confirmation through in vivo experiments aimed at determining whether GATA4 overexpression leads to vascular relaxation.

In conclusion, the results of the present study indicated that GATA4 may be a transcription factor that induces NOX4 expression, which results in increased production of NO. Additionally, GATA4 inhibited hyperglycemia-induced endothelial dysfunction in both HUVECs and a mouse model of diabetes. This protective effect of GATA4 was experimentally linked to increased production of NO and p-eNOS. Finally, the protection of endothelial function by simvastatin treatment was demonstrated to be achieved through GATA4 expression. Overall, the present findings revealed a novel molecular mechanism in endothelial dysfunction and identified GATA4 as a potential therapeutic target for preventing endothelial dysfunction in diabetes patients.

\section{Acknowledgements}

This study was supported by The Natural Science Foundation of Zhejiang Province (grant no. LZ16H020001) and The National Natural Science Foundation of China (grant no. 81170167).

\section{References}

1. Mellbin LG, Anselmino M and Rydén L: Diabetes, prediabetes and cardiovascular risk. Eur J Cardiovasc Prev Rehabil 17 (Suppl 1): S9-S14, 2010.

2. Riddle MC: Glycemic control and cardiovascular mortality. Curr Opin Endocrinol Diabetes Obes 18: 104-109, 2011.

3. Bentzon JF, Otsuka F, Virmani R and Falk E: Mechanisms of plaque formation and rupture. Circ Res 114: 1852-1866, 2014.

4. Sun Z: Atherosclerosis and atheroma plaque rupture: Normal anatomy of vasa vasorum and their role associated with atherosclerosis. ScientificWorldJournal 2014: 285058, 2014.

5. Polovina MM and Potpara TS: Endothelial dysfunction in metabolic and vascular disorders. Postgrad Med 126: 38-53, 2014.

6. Bondarenko PG and Sil'Chenko TS: Status of the immune system in peptic ulcer patients before and after vagotomy. Klin Khir: 27-29, 1983 (In Russian).

7. Langbein H, Brunssen C, Hofmann A, Cimalla P, Brux M, Bornstein SR, Deussen A, Koch E and Morawietz H: NADPH oxidase 4 protects against development of endothelial dysfunction and atherosclerosis in LDL receptor deficient mice. Eur Heart J 37: 1753-1761, 2016.

8. Kleinschnitz C, Grund H, Wingler K, Armitage ME, Jones E, Mittal M, Barit D, Schwarz T, Geis C, Kraft P, et al: Post-stroke inhibition of induced NADPH oxidase type 4 prevents oxidative stress and neurodegeneration. PLoS Biol 8: pii: e1000479, 2010.

9. Kuroda J, Ago T, Matsushima S, Zhai P, Schneider MD and Sadoshima J: NADPH oxidase 4 (Nox4) is a major source of oxidative stress in the failing heart. Proc Natl Acad Sci USA 107: 15565-15570, 2010.

10. Ray R, Murdoch CE, Wang M, Santos CX,Zhang M,Alom-Ruiz S, Anilkumar N, Ouattara A, Cave AC, Walker SJ, et al: Endothelial Nox4 NADPH oxidase enhances vasodilatation and reduces blood pressure in vivo. Arterioscler Thromb Vasc Biol 31: 1368-1376, 2011.
11. Manea SA, Todirita A, Raicu M and Manea A: C/EBP transcription factors regulate NADPH oxidase in human aortic smooth muscle cells. J Cell Mol Med 18: 1467-1477, 2014.

12. Manea A, Tanase LI, Raicu M and Simionescu M: Jak/STAT signaling pathway regulates nox1 and nox4-based NADPH oxidase in human aortic smooth muscle cells. Arterioscler Thromb Vasc Biol 30: 105-112, 2010.

13. Sun Z, Han J, Zhao W, Zhang Y, Wang S, Ye L, Liu T and Zheng L: TRPV1 activation exacerbates hypoxia/reoxygenation-induced apoptosis in H9C2 cells via calcium overload and mitochondrial dysfunction. Int J Mol Sci 15: 18362-18380, 2014.

14. Zhao C, Guo H, Li J, Myint T, Pittman W, Yang L, Zhong W, Schwartz RJ, Schwarz JJ, Singer HA, et al: Numb family proteins are essential for cardiac morphogenesis and progenitor differentiation. Development 141: 281-295, 2014.

15. Zhong X, Xiu LL, Wei GH, Liu YY, Su L, Cao XP, Li YB and Xiao HP: Bezafibrate enhances proliferation and differentiation of osteoblastic MC3T3-E1 cells via AMPK and eNOS activation. Acta Pharmacol Sin 32: 591-600, 2011.

16. Hou HH, Liao YJ, Hsiao SH, Shyue SK and Lee TS: Role of phosphatase activity of soluble epoxide hydrolase in regulating simvastatin-activated endothelial nitric oxide synthase. Sci Rep 5: 13524, 2015.

17. Murray TV, Smyrnias I, Shah AM and Brewer AC: NADPH oxidase 4 regulates cardiomyocyte differentiation via redox activation of c-Jun protein and the cis-regulation of GATA-4 gene transcription. J Biol Chem 288: 15745-15759, 2013.

18. Tremblay JJ, Hamel F and Viger RS: Protein kinase A-dependent cooperation between GATA and CCAAT/enhancer-binding protein transcription factors regulates steroidogenic acute regulatory protein promoter activity. Endocrinology 143: 3935-3945, 2002.

19. LaVoie HA, Singh D and Hui YY: Concerted regulation of the porcine steroidogenic acute regulatory protein gene promoter activity by follicle-stimulating hormone and insulin-like growth factor I in granulosa cells involves GATA-4 and CCAAT/enhancer binding protein beta. Endocrinology 145: 3122-3134, 2004.

20. Broderick TL, Parrott CR, Wang D, Jankowski M and Gutkowska J: Expression of cardiac GATA4 and downstream genes after exercise training in the $\mathrm{db} / \mathrm{db}$ mouse. Pathophysiology 19: 193-203, 2012.

21. Broderick TL, Jankowski M, Wang D, Danalache BA, Parrott CR and Gutkowska J: Downregulation in GATA4 and downstream structural and contractile genes in the $\mathrm{db} / \mathrm{db}$ mouse heart. ISRN Endocrinol 2012: 736860, 2012.

22. Shaw-Smith C, De Franco E, Lango Allen H, Batlle M, Flanagan SE, Borowiec M, Taplin CE, van Alfen-van der Velden J, Cruz-Rojo J, Perez de Nanclares G, et al: GATA4 mutations are a cause of neonatal and childhood-onset diabetes. Diabetes 63: 2888-2894, 2014.

23. Muiya NP, Wakil SM, Tahir AI, Hagos S, Najai M, Gueco D, Al-Tassan N, Andres E, Mazher N, Meyer BF and Dzimiri N: A study of the role of GATA4 polymorphism in cardiovascular metabolic disorders. Hum Genomics 7: 25, 2013.

24. Vaskivuo TE, Anttonen M, Herva R, Billig H, Dorland M, te Velde ER, Stenbäck F, Heikinheimo M and Tapanainen JS: Survival of human ovarian follicles from fetal to adult life: Apoptosis, apoptosis-related proteins, and transcription factor GATA-4. J Clin Endocrinol Metab 86: 3421-3429, 2001.

25. Li HX, Zhou YF, Zhao X, Jiang B and Yang XJ: GATA-4 protects against hypoxia-induced cardiomyocyte injury: Effects on mitochondrial membrane potential. Can J Physiol Pharmacol 92: 669-678, 2014.

26. Bielli A, Scioli MG, Mazzaglia D, Doldo E and Orlandi A: Antioxidants and vascular health. Life Sci 143: 209-216, 2015.

27. Aries A, Paradis P, Lefebvre C, Schwartz RJ and Nemer M: Essential role of GATA-4 in cell survival and drug-induced cardiotoxicity. Proc Natl Acad Sci USA 101: 6975-6980, 2004.

28. Kobayashi S, Lackey T, Huang Y, Bisping E, Pu WT, Boxer LM and Liang Q: Transcription factor gata4 regulates cardiac BCL2 gene expression in vitro and in vivo. FASEB J 20: 800-802, 2006.

This work is licensed under a Creative Commons Attribution-NonCommercial-NoDerivatives 4.0 International (CC BY-NC-ND 4.0) License. 